\title{
Semiconductor Surface Science and Technology
}

\section{EIW-7, Europhysics Industrial Workshop}

\author{
Leningrad, 19-22 June 1990
}

We feel that it is important to publish this report on the EIW-7, which is long overdue owing to other commitments, because the information on Institutes in the USSR is of general interest. We apologize to all concerned for the delay.

The first meeting of this kind was very successful. About 45 people participated, of which 20 were from The Netherlands, the UK, Germany, Italy, Hungary, Czechoslovakia and Switzerland with the balance from 12 different Russian institutes (in Leningrad, Moscow, Kazan and Novosibirsk) representing mainly the larger research institutes such as branches of the Academy of Science. Only a few came from purely scientific university groups and from truly industrial organizations.

Although the emphasis of the meeting was on silicon, several papers dealt with $\mathrm{GaAs}$ compounds. The subjects were:
J. Kistemaker (left) and F.W. Saris, the organizers of EIW-7.
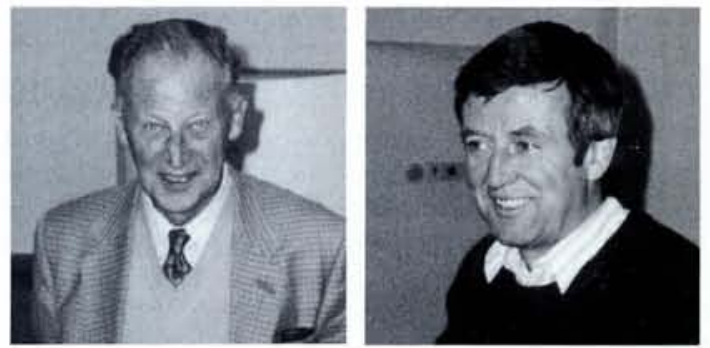

submicron lithography, ion implantation, silicon-metal compounds, plasma-processes and instrumentation, molecular beam epitaxy, gallium arsenide devices, electrical and optical characterization and power devices. They were spread over 25 lectures, all with simultaneous translation, and there was plenty of time for questions and discussion.

In the afternoon of 21 st June, the group visited the Instrument Division of the Science \& Technology Corporation in Leningrad where about 300 people, mainly scientists and engineers, work.

The local committee (M.L. Alexandrov, A. Berezin and V. Markov) were our hosts for the evening programmes in which we not only attended the re-opening of the
Smolny Cathedral with a Rachmaninov concert, but also enjoyed a boat trip by night on the River Neva.

Contributions by the Institutes described below should be mentioned as they USSR, all sponsored by the Russian Academy of Sciences.

We think that the initiative of the European Physical Society and the Science and Technology Corporation in Leningrad to promote liks between researchers in the universities and in the industrially orientated centres via the workshop was constructive and should be continued.

\section{J. Kistemaker, F.W. Saris} FOM-Institute Amolf, Amsterdam represent leading research groups in the

\section{Semiconductor Research in the USSR}

1. A.F. Joffe Physico-Technical Institute - Semiconductor Division

Polytechnicheskaya 26, 194219 Leningrad

The Director (Academician Zh.l. Alferov) was recently been nominated Vice-President of the Academy of Sciences for microelectronic devices. The Institute presented work at EIW-7 on:

- Radiative recombinations in quantumhereterostructures. Yields of $1.4 \%$ have been achieved and the target is $6 \%$ (D. Garbusov, S. Gurevich).

- Molecular beam epitaxy (P. Kopiev). - Space-charges in irradiated semiconductors (P. Koper together with V. Korablev of the Politechnical Institute which is annexed to the Joffe Institute).

\section{Institute of Solid State Physics}

V.A. Grazhulis is the Deputy Director but in fact he replaces Academician Y. Ossipyan as Director. Professor Grazhulis is working on semiconductor surfaces and molecular beam epitaxy.

\section{Institutes of Physics and Technology} Krusikov St. 25a, 117218 Moscow

This Institute, whose Director is Academician K.A. Valiev, is an offspring of the famous Lebedev Institute and is located close by. Members of the Institute reported at EIW-7 on:

- High precision lithography done by optical means with a (KrF) excimer laser (K. Valiev who is also Secretary of the Division for informatics, computational techniques and automation of the Academy of Sciences).

- Superstructures to be determined by forward and/or backward reflected Bragg patterns of $\mathrm{Cu}-\mathrm{K}_{\alpha}$ radiation (A.M. Afanaseyev, formerly at the Kurchatov Institute). - Growth of crystalline $\mathrm{TiSi}_{2}$ layers, 50 $\mathrm{nm}$ below the surface of a (111) Si crystal by implantation of $100 \mathrm{keV} \mathrm{Ti}+$ ions (A.A. Orlikovskij).

- Acoustic analyses of internal structures of microelectronic materials (V.N. Repin and D.A. Sharov).

\section{Institute of Microelectronics Techno-} logy and High Purity Materials 142432 Chernogolovka, Moscow District

This Institute if located next to the Institute of Solid State Physics of which it is an offspring.

The Director (Prof. V.V. Artistov) specializes in the UPS of metal-semiconductor interfaces and low energy electron loss spectroscopy (LEELS) of $\mathrm{Ag} / \mathrm{Si}, \mathrm{Ge}, \mathrm{A}_{3} \mathrm{~B}_{5}$, in combination with LEED and AES.

The Deputy Director (Prof. A. Viatkin) leads a group working on ion beam modification and analysis of semiconductors. Together with $\mathrm{Y}$. Yerochin he is studying ion-assisted solid phase epitaxy of $\mathrm{Si}$ and Ge. They possess a MEIS system from HVEE together with a $500 \mathrm{keV}$ implanter.

S. Ju. Shapoval (Head of the Epitaxy Laboratory) has developed an atomic hydrogen source (ECR-type) for the creation of high density ion and radical fluxes when etching, cleaning and depositing on semiconductor, dielectric, superconducting and other material surfaces. This system is commercially available.

A.A. Snigirev (Head of the $X$-Ray Crystal Optics Group) has developed Fresnel-
Bragg lenses and micro-zone plates for $\mathrm{X}$-ray imaging. The Group makes use of facilities in Hamburg and Orsay.

\section{Other Activities}

- Institute of Physical Chemistry (Moscow): B.V. Spitzyn grows doped (B) diamond.

- Institute of Semiconductor Physics (Novosibirsk): S.J. Stenin works on Si-Ge and on In-As interfaces.

- Institutes of Radio Engineering and Electronics (Moscow): V.G. Mokerov works together with the Max-Planck Institute in Stuttgart on fast delta-alloyed transistors (GaAs).

- Kazan Physico-Technical Institute: J.B. Khaibullin leads research on the modification of semiconductor, metal and optical surfaces by means of ion beams.

One should note that these various research institutes mostly belong to the Academy or to universities. There are also many laboratories and institutes which form part of the Ministry for Electronics, but they have little contact with the academic world.

\section{R. and D. Collaboration in Central Europe}

The 1991 Meeting of the EPS Associate Members will be held on Friday, 20 September in Cracow, Poland immediately after the EPS sponsored "Physics for Industry - Industry for Physics" conference. Presentations and a round-table discussion on $R$. and D. collaboration in Central Europe will be followed by meetings with experts and regional authorities and a short social programme. 